Pacific Journal of Mathematics

CONGRUENCE LATTICES OF SEMILATTICES

S. FreEs AND JaMES BRYANT Nation 


\title{
CONGRUENCE LATTICES OF SEMILATTICES
}

\author{
Ralph Freese and J. B. Nation
}

\begin{abstract}
The main result of this paper is that the class of congruence lattices of semilattices satisfies no nontrivial lattice identities. It is also shown that the class of subalgebra lattices of semilattices satisfies no nontrivial lattice identities. As a consequence it is shown that if $\mathscr{Y}$ is a semigroup variety all of whose congruence lattices satisfy some fixed nontrivial lattice identity, then all the members of $\mathscr{C}$ are groups with exponent dividing a fixed finite number.
\end{abstract}

Given a variety (equational class) $\mathscr{K}$ of algebras, among the interesting questions we can ask about the members of $\mathscr{K}$ is the following: does there exist a lattice identity $\delta$ such that for each algebra $A \in \mathscr{K}$, the congruence lattice $\Theta(A)$ satisfies $\delta$ ? In the case that $\mathscr{K}$ has distributive congruences, many strong conclusions can be drawn about the algebras of $\mathscr{K}[1,2,7]$. In the case that $\mathscr{C}$ has permutable congruences or modular congruences, there is reason to hope that some similar results may be obtainable $[4,8]$.

A standard method of proving that a class of lattices satisfies no nontrivial lattice identities is to show that all partition lattices (lattices of equivalence relations) are contained as sublattices. The lattices of congruences of semilattices, however, are known to be pseudo-complemented [9]. It follows that the partition lattice on a three-element set (the five-element two-dimensional lattice) is not isomorphic to a sublattice of the congruence lattice of a semilattice, and in fact is not a homomorphic image of a sublattice of the congruence lattice of a finite semilattice. Nonetheless we shall show in this paper that the congruence lattices of semilattices satisfy no nontrivial lattice identities. This solves Problem 6 of [10]. Using a theorem of T. Evans [6], we also show that if $\mathscr{Y}$ is a variety of semigroups all of whose congruence lattices satisfy some fixed nontrivial lattice identity, then all the members of $\mathscr{f}$ are groups with exponent dividing a fixed finite number.

In $\S 1$ we give definitions and a few basic facts about the congruences of semilattices. In $\S 2$ we prove our main theorem, and in $\S 3$ we apply it to obtain the corollary about varieties of semigroups.

1. A semilattice is a commutative idempotent semigroup. We may impose a partial ordering on a semilattice $S$ by defining

$$
x \leqq y \quad \text { if } \quad x y=x .
$$


Under this ordering, any two elements $x, y \in S$ have a greatest lower bound, namely their product $x y$. $S$ is called a meet semilattice. It may be that $x$ and $y$ have a least upper bound $w \in S$; if so, we define

$$
x+y=w \text {. }
$$

Thus + is a partial operation on $S$, and $x+y$ is called the join of $x$ and $y$. If $S$ is finite, and if $x$ and $y$ have a common upper bound, then $x+y$ exists and

$$
x+y=\Pi\{z \in S: z \geqq x \text { and } z \geqq y\} .
$$

The least element of a semilattice, if it exists, is denoted by 0 ; the greatest element, if it exists, by 1.

A dual ideal of a semilattice $S$ is a set $D \cong S$ satisfying (i) $d_{1}, d_{2} \in D$ implies $d_{1} d_{2} \in D$, and (ii) $x \geqq d \in D$ implies $x \in D$. We will denote the principal dual ideal above $x$ by $1 / x$, i.e.,

$$
1 / x=\{z \in S: z \geqq x\} \text {. }
$$

For reference we note that if $x+y$ is defined, then

$$
1 / x \cap 1 / y=1 / x+y \text {. }
$$

If $S$ and $T$ are semilattices, then $S \times T$ will denote the (external) direct product of $S$ and $T$. We shall use round symbols $(\boldsymbol{\Lambda}, \mathbf{U})$ for set operations, and sharp symbols $(\Lambda, V)$ for lattice operations.

The following theorem is basic to the study of semilattice congruences.

Theorem 1. [9] Let 2 denote the two-element semilattice. If $S$ is any semilattice and $D$ is a dual ideal of $S$, then the mapping $f: S \rightarrow 2$ defined by

$$
f(x)=\left\{\begin{array}{lll}
1 & \text { if } & x \in D \\
0 & \text { if } & x \notin D
\end{array}\right.
$$

is a homomorphism. Thus 2 is the only subdirectly irreducible semilattice, and the dual of $\Theta(S)$ is a point lattice $(\Theta(S)$ is a copoint lattice).

In the rest of this section we note some easily provable facts about the congruence lattice of a semilattice $S$.

(1) Suppose $\Theta(S)$ is atomic, and let $A$ be the set of atoms of $\Theta(S)$. Let $x^{*}$ denote the pseudo-complement of $x$. Then if $a \in A, a^{*}$ is a coatom of $\Theta(S)$, and 0 is a unique irredundant meet of $\left\{a^{*}: a \in A\right\}$. Hence $S$ is a unique subdirect product of $|A|$ copies of 2 , but of no fewer. 
It is not hard to show that if $\theta$ covers 0 in $\Theta(S)$ then $\theta$ covers 0 in $\Pi(S)$, the partition lattice on $S$. From this and Theorem 1 it follows that

(2) $\Theta(S)$ is semimodular and if $\Theta(S)$ is finite and $c$ is the number of coatoms of $\Theta(S)$ then

$$
\operatorname{dim} \Theta(S)=c=|S|-1 .
$$

(3) $\Theta(S)$ is relatively pseudo-complemented [9].

A lattice $L$ is called locally distributive if the quotient sublattices $u_{a} / a$ is distributive for all $a \in L$, where $u_{a}$ is the join of the elements covering $a$. In a compactly generated lattice, local distributivity is equivalent to the conjunction of semimodularity and relatively pseudocomplementation [3]. Hence

(4) $\Theta(S)$ is locally distributive.

The problem of characterizing all lattices isomorphic to congruence lattices of semilattices remains open. The above conditions are not sufficient, even in the finite case.

2. In this section we prove the main result of this paper.

THEOREM 2. Let $\delta$ be a nontrivial lattice identity. Then there exists a finite semilattice $S(\delta)$ such that $\delta$ fails in the congruence lattice $\Theta(S(\delta))$.

The theorem is an immediate consequence of Lemmas 1 and 4 to be proven below.

Lemma 1. Let $S$ be a finite meet semilattice, and let $\mathscr{S}(S)$ be the lattice of (partial) join-subalgebras of $S$, with $0 \in S$ considered as a distinguished element. Then the congruence lattice $\Theta(S)$ is dually isomorphic to $\mathscr{S}(S)$.

A partial join subalgebra of $S$ is a subset containing 0 and closed under joins, whenever they exist.

Proof. The dual atoms of $\theta(S)$ are the partitions $\psi_{d}=(1 / d)$ $(S-1 / d)$ for $0 \neq d \in S$. On the other hand, the atoms of $\mathscr{S}(S)$ are the subalgebras $\xi_{d}=\{0, d\}$ for $0 \neq d \in S$. We want to show that the mapping $\psi_{d} \rightarrow \xi_{d}$ induces a dual isomorphism of $\theta(S)$ onto $\mathscr{S}(S)$. Since $\Theta(S)$ is a copoint lattice and $\mathscr{S}(S)$ is a point lattice, it is sufficient to show that their closure operations are duals under the mapping, i.e., that

$$
\psi_{c} \geqq \psi_{d_{1}} \wedge \cdots \wedge \psi_{d_{k}} \text { if and only if } \xi_{c} \leqq \xi_{d_{1}} \vee \cdots \vee \xi_{d_{k}} .
$$


This is equivalent to

$$
\psi_{c} \geqq \psi_{d_{1}} \wedge \cdots \wedge \psi_{d_{k}} \text { if and only if } c \in\left\langle d_{1}, \cdots, d_{k}\right\rangle
$$

where $\left\langle d_{1}, \cdots, d_{k}\right\rangle$ denotes the join subalgebra generated by $\left\{d_{1}, \cdots, d_{k}\right\}$. Notice that the equivalence classes of $\psi_{d_{1}} \wedge \cdots \wedge \psi_{d_{k}}$ are

$$
\left(\bigcap_{i \in I} 1 / d_{i}-\bigcup_{j \in I^{c}} 1 / d_{j}\right)
$$

for $I \leqq\{1, \cdots, k\}$. If $\psi_{c} \geqq \psi_{d_{1}} \wedge \cdots \wedge \psi_{d_{k}}$ then each of these classes is contained in either $1 / c$ or $S-1 / c$. Considered the $\psi_{d_{1}} \wedge \cdots \wedge \psi_{d_{k}}$ class which contains $c$. Then $c$ is the least element of that class, and thus

$$
c=\sum_{i \in I} d_{i} \text { for some } I \subseteq\{1, \cdots, k\} \text {. }
$$

Hence $c \in\left\langle d_{1}, \cdots, d_{k}\right\rangle$.

Conversely, if $c \in\left\langle d_{1}, \cdots, d_{k}\right\rangle$, then $c=\sum_{i \in I} d_{i}$ for some $I \subseteq$ $\{1, \cdots, k\}$. Thus the congruence $\Lambda_{i \in I} \psi_{d_{i}}$ has one class equal to $1 / c$ and the rest contained in $S-1 / c$. Hence

$$
\psi_{c} \geqq \bigwedge_{i \in I} \psi_{d_{i}} \geqq \psi_{d_{1}} \wedge \cdots \wedge \psi_{d_{k}}
$$

This completes the proof of Lemma 1 .

Suppose $\sigma \leqq \tau$ is a nontrivial lattice identity, i.e., $\sigma \leqq \tau$ does not hold in a free lattice. Then we construct a finite semilattice $S(\sigma)$ (depending only on $\sigma$ ) such that $\sigma \leqq \tau$ fails in $\mathscr{S}(S(\sigma))$. Combined with Lemma 1, this will prove Theorem 2.

Let $X=\{x, y, z, \cdots\}$ be a countable set, and let $F L(X)$ donote the free lattice on $X$. For each element $\sigma \in F L(X)$ we will define a finite semilattice $S(\sigma)$. First of all we write each $\sigma \in F L(X)$ in canonical form. Then we define

$$
\begin{aligned}
S(x) & =\underset{\sim}{2} \quad \text { for } x \in X \\
S\left(\sigma_{1} \vee \sigma_{2}\right) & =S\left(\sigma_{1}\right) \times S\left(\sigma_{2}\right) \\
S\left(\sigma_{1} \wedge \sigma_{2}\right) & =S\left(\sigma_{1}\right) \times S\left(\sigma_{2}\right)-\Gamma
\end{aligned}
$$

where

$$
\Gamma=1 /(1,0) \cup 1 /(0,1)-\{(1,1)\} .
$$

Let us look more carefully at the construction. If $S\left(\sigma_{1}\right)$ and $S\left(\sigma_{2}\right)$ are lattices, then $S\left(\sigma_{1}\right) \times S\left(\sigma_{2}\right)-\Gamma$ is meet-closed and has a unit element; hence it is a lattice. It follows by induction that $S(\sigma)$ is a lattice for each $\sigma \in F L(X)$. We need to know how to compute joins in $S(\sigma)$. In $S\left(\sigma_{1} \vee \sigma_{2}\right)$ joins are of course taken componentwise. In $S\left(\sigma_{1} \wedge \sigma_{2}\right)$ we have 
$(*) \quad\left(r_{1}, r_{2}\right)+\left(s_{1}, s_{2}\right)$

$$
=\left\{\begin{array}{lll}
\left(r_{1}+s_{1}, r_{2}+s_{2}\right) & \text { if } \quad r_{1}+s_{1} \neq 1 \quad \text { and } r_{2}+s_{2} \neq 1 \\
(1,1) & \text { if } \quad r_{1}+s_{1}=1 \quad \text { or } \quad r_{2}+s_{2}=1
\end{array}\right.
$$

In any $S(\sigma)$ let us denote $(1,1)$ by 1 .

For each $\sigma \in F L(X)$ we now define a homomorphism $\varphi_{\sigma}$ of $F L(X)$ into $\mathscr{S}(S(\sigma))$. We do this by associating with each $y \in X$ a join-subalgebra $\varphi_{\sigma}(y)$ of $S(\sigma)$, and extending this map to a homomorphism in the (unique) natural way. Once again we proceed inductively, with $\sigma \in F L(X)$ written in canonical form. For $y \in X$ we set

$$
\begin{aligned}
& \varphi_{x}(y)= \begin{cases}S(x) & \text { if } \quad y=x \\
\{0\} & \text { if } \quad y \neq x\end{cases} \\
& \varphi_{\sigma_{1} \vee \sigma_{2}}(y)=\left\{\left(r_{1}, r_{2}\right): r_{1} \in \varphi_{\sigma_{1}}(y), r_{2} \in \varphi_{\sigma_{2}}(y)\right\} \\
& \varphi_{\sigma_{1} \wedge \sigma_{2}}(y)=\left\{\left(r_{1}, r_{2}\right): r_{1} \in \varphi_{\sigma_{1}}(y)-\{1\}, r_{2} \in \varphi_{\sigma_{2}}(y)-\{1\}\right\} \\
& \cup \cup\left(\varphi_{\sigma_{1}}(y), \varphi_{\sigma_{2}}(y)\right)
\end{aligned}
$$

where

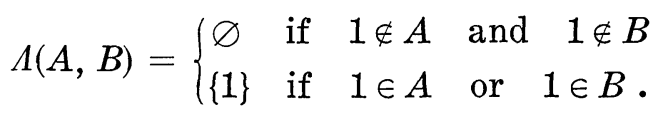

Our computations will be based upon the following lemma.

Lemma 2. If $\rho \in F L(X)$, then

(i) $\varphi_{\sigma_{1} \vee \sigma_{2}}(\rho)=\varphi_{\sigma_{1}}(\rho) \times \varphi_{\sigma_{2}}(\rho)$

(ii) $\varphi_{\sigma_{1} \wedge \sigma_{2}}(\rho)-\{1\}=\left\{(r, s) \in \varphi_{\sigma_{1}}(\rho) \times \varphi_{o_{2}}(\rho): r \neq 1\right.$ and $\left.s \neq 1\right\}$.

Proof. We prove (ii); the proof of (i) is similar but easier. We proceed by induction on the length of $\rho$. For $\rho=y \in X$ the lemma is immediate from the definitions. Now note that since $0 \in T$ for every $T \in \mathscr{S}(S(\sigma))$, we have

$$
T_{1} \vee T_{2}=\left\{t_{1}+t_{2}: t_{1} \in T_{1}, t_{2} \in T_{2}\right\} \text {. }
$$

Hence if $\rho=\rho_{1} \vee \rho_{2}$, then by $(*)$ we have

$$
\begin{aligned}
\varphi_{\sigma_{1} \wedge \sigma_{2}}(\rho)-\{1\}= & \varphi_{\sigma_{1} \wedge \sigma_{2}}\left(\rho_{1}\right) \vee \varphi_{\sigma_{1} \wedge \sigma_{2}}\left(\rho_{2}\right)-\{1\} \\
= & \left\{\left(r_{1}, s_{1}\right)+\left(r_{2}, s_{2}\right):\left(r_{1}, s_{1}\right) \in \varphi_{\sigma_{1} \wedge \sigma_{2}}\left(\rho_{1}\right),\right. \\
& \left.\left(r_{2}, s_{2}\right) \in \varphi_{\sigma_{1} \wedge \sigma_{1}}\left(\rho_{2}\right), r_{1}+r_{2} \neq 1, s_{1}+s_{2} \neq 1\right\} .
\end{aligned}
$$

By the inductive hypothesis we have

$$
\left(r_{i}, s_{i}\right) \in \varphi_{\sigma_{1} \wedge \sigma_{2}}\left(\rho_{i}\right)-\{1\}=\varphi_{\sigma_{1}}\left(\rho_{i}\right)-\{1\} \times \varphi_{\sigma_{2}}\left(\rho_{i}\right)-\{1\}
$$

for $i=1,2$ and hence 


$$
\varphi_{\sigma_{1} \wedge \sigma_{2}}(\rho)-\{1\}=\left\{(r, s) \in \varphi_{\sigma_{1}}(\rho) \times \varphi_{\sigma_{2}}(\rho): r \neq 1 \text { and } s \neq 1\right\} .
$$

On the other hand, if $\rho=\rho_{1} \wedge \rho_{2}$, then

$$
\varphi_{\sigma_{1} \wedge \sigma_{2}}(\rho)-\{1\}=\varphi_{\sigma_{1} \wedge \sigma_{2}}\left(\rho_{1}\right)-\{1\} \cap \varphi_{\sigma_{1} \wedge \sigma_{2}}\left(\rho_{2}\right)-\{1\}
$$

and the conclusion of the lemma follows.

LEMma 3. If $\rho \in F L(X)$, then $1 \in \varphi_{\rho}(\rho)$.

Proof. As usual we proceed by induction on the length of $\rho$. If $\rho=y \in X$ the lemma follows from the definitions. If $\rho=\rho_{1} \vee \rho_{2}$, then $1 \in \varphi_{\rho_{i}}\left(\rho_{i}\right)(i=1,2)$, and thus by Lemma 2 (i) we have

$$
(1,0) \in \varphi_{\rho}\left(\rho_{1}\right) \text { and }(0,1) \in \varphi_{\rho}\left(\rho_{2}\right)
$$

from which it follows that $1 \in \varphi_{\rho}\left(\rho_{1}\right) \vee \varphi_{\rho}\left(\rho_{2}\right)=\varphi_{\rho}(\rho)$.

If $\rho=\rho_{1} \wedge \rho_{2}$, we can again assume $1 \in \rho_{\rho_{i}}\left(\rho_{i}\right)$ for $i=1,2$. We need to show that $1 \in \varphi_{\rho_{1} \wedge \rho_{2}}\left(\rho_{i}\right)$. We prove a slightly stronger statement: if $1 \in \varphi_{\rho_{1}}(\sigma)$, then $1 \in \varphi_{\rho_{1} \wedge \rho_{2}}(\sigma)$. If $\sigma=y \in X$ this is immediate. Suppose $\sigma=\sigma_{1} \vee \sigma_{2}$, then $1 \in \varphi_{\rho_{1}}\left(\sigma_{1}\right) \vee \varphi_{\rho_{1}}\left(\sigma_{2}\right)$ and hence $1=t_{1}+t_{2}$, where $t_{i} \in \varphi_{\rho_{1}}\left(\sigma_{i}\right)$. If $t_{1} \neq 1, t_{2} \neq 1$, then by Lemma 2 (ii) we have

$$
1=\left(t_{1}, 0\right)+\left(t_{2}, 0\right) \in \varphi_{\rho_{1} \wedge \rho_{2}}\left(\sigma_{1}\right) \vee \varphi_{\rho_{1} \wedge \rho_{2}}\left(\sigma_{2}\right)=\varphi_{\rho_{1} \wedge \rho_{2}}(\sigma) .
$$

If $t_{i}=1$ for some $i$ then by induction $1 \in \varphi_{\rho_{1}}\left(\sigma_{i}\right)$ implies

$$
1 \in \varphi_{\rho_{1} \wedge \rho_{2}}\left(\sigma_{i}\right) \cong \varphi_{\rho_{1} \wedge \rho_{2}}(\sigma) \text {. }
$$

Suppose $\sigma=\sigma_{1} \wedge \sigma_{2}$. Then $1 \in \varphi_{\rho_{1}}\left(\sigma_{1}\right) \cap \varphi_{\rho_{1}}\left(\sigma_{2}\right)$. By induction $1 \in$ $\varphi_{\rho_{1} \wedge \rho_{2}}\left(\sigma_{i}\right)$ for $i=1,2$ and we are done.

Lemma 4. If $\sigma \not \leq \tau$ in $F L(X)$, then $1 \notin \varphi_{o}(\tau)$.

Assume we have proven Lemma 4. Then Lemmas 3 and 4 combine to yield: $1 \in \varphi_{\sigma}(\tau)$ if and only if $\sigma \leqq \tau$ in $F L(X)$. Hence $\varphi_{\sigma}(\sigma) \subseteq$ $\varphi_{\sigma}(\tau)$ if and only if $\sigma \leqq \tau$ in $F L(X)$, and Theorem 2 follows.

Proof of Lemma 4. Suppose the lemma is false. Let $\sigma$ be a word of minimum length such that $1 \in \varphi_{o}\left(\tau^{\prime}\right)$ for some $\tau^{\prime}$ such that $\sigma \not \tau^{\prime}$ in $F L(X)$. Let $\tau$ be of minimal length such that $\sigma \not \tau$ and $1 \in \Phi_{\sigma}(\tau)$. We will show that these conditions lead to a contradiction. The cases $\sigma \in X, \sigma=\sigma_{1} \vee \sigma_{2}, \sigma=\sigma_{1} \wedge \sigma_{2}$, and $\tau \in X$ or $\tau=\tau_{1} \wedge \tau_{2}$ are easy to handle. Let us assume, then, that $\sigma=\sigma_{1} \wedge \sigma_{2}$ and $\tau=\tau_{1} \vee \tau_{2}$. Then since $\sigma \leqq \tau$ we have

$$
\sigma \leqq \tau_{1} \text { and } \sigma \leqq \tau_{2} \text { and } \sigma_{1} \not \tau \text { and } \sigma_{2} \leqq \tau \text {. }
$$


Since $1 \in \varphi_{\sigma}(\tau)=\varphi_{\sigma}\left(\tau_{1}\right) \vee \varphi_{\sigma}\left(\tau_{2}\right)$, there exist $t_{i} \in \varphi_{\sigma}\left(\tau_{i}\right)$ such that $t_{1}+t_{2}=1$. If $t_{i}=1$ for some $i$ then by the minimal length of $\tau$ we have $\sigma \leqq \tau_{i}$, a contradiction. Thus $t_{i} \neq 1$ and by Lemma 2 (ii) we can write $t_{i}=\left(r_{i}, s_{i}\right)$ where $r_{i} \in \varphi_{\sigma_{1}}\left(\tau_{i}\right)$ and $s_{i} \in \varphi_{\tau_{2}}\left(\tau_{i}\right)$. Now either $r_{1}+r_{2}=1$ in $S\left(\sigma_{1}\right)$, which means $1 \in \varphi_{\sigma_{1}}\left(\tau_{1}\right) \vee \varphi_{\sigma_{1}}\left(\tau_{2}\right)=\varphi_{\sigma_{1}}(\tau)$ and hence $\sigma_{1} \leqq \tau$, or $s_{1}+s_{2}=1$ and $\sigma_{2} \leqq \tau$. Both these statements are contradictions.

Since the semilattices $S(\sigma)$ constructed above are in fact lattices, they are join semilattices. Thus, the above proof shows that any nontrivial lattice identity fails in the subalgebra lattice of some finite semilattice.

Now the congruence lattices of lattices satisfy every nontrivial lattice identity, while those of semilattices satisfy no identity. It is reasonable then to ask if there is some "natural" restricted class $\mathscr{K}$ of semilattices such that the congruence lattices of semilattices in $\mathscr{r}$ satisfy some lattice identity.

One such class is known [5]. A simple argument based on Theorem 1 shows that $\theta(S)$ is nonmodular if and only if $S$ contains a pair of noncomparable elements with a common upper bound. Hence if $S$ is finite $\theta(S)$ is either nonmodular, or else it is isomorphic to the Boolean algebra of subsets of some set.

Ot the other hand, the semilattices $S(\sigma)$ constructed in $\S 2$ are in fact lattices; in particular, the join of every pair of elements is defined. It follows from Theorem 1 that $S(\sigma)$ can be imbedded as a join semilattice into a Boolean algebra $B(\sigma)$. Considering $B(\sigma)$ as a meet semilattice, we see that every nontrivial lattice identity fails in the (semilattice) congruence of some finite Boolean algebra.

3. We can now prove an interesting corollary about varieties of semigroups. Let $R$ denote the two-element semigroup with multiplication law $x y=y ; L$ the two-element semigroup with multiplication law $x y=x$; and $C$ the two-element semigroup with constant multiplication. The following theorem is due to T. Evans [6].

THEOREM 3. The atoms of the lattice of varieties of semigroups are the varieties generated by $R, L, C, \underset{\sim}{2}$ (the variety of all semilattices), and the cyclic groups of prime order. If a nontrivial variety of semigroups does not contain $R, L, C$, or $\underset{\sim}{2}$, then it is a subvariety of $\mathscr{B}_{n}$, the variety of groups of exponent dividing $n$, for some finite $n$.

Now if $T$ is a semigroup in the variety generated by $R, L$, or $C$, then $\Theta(T)$ is just the partition lattice on $T$. Hence Theorems 2 and 3 combine to give the following corollary.

CoROLlaRY. If of is a semigroup variety all of whose congruence 
lattices satisfy some fixed nontrivial lattice identity, then or is a subvariety of $\mathscr{B}_{n}$ for some finite $n$.

\section{REFERENCES}

1. K. A. Baker, Equational bases for finite algebras, Notices Amer. Math. Soc., 19 (1972), A-44.

2. - Primitive satisfaction and equational problems for lattices and other algebras, to appear.

3. P. Crawley and R. P. Dilworth, Algebraic Theory of Lattices, Prentice-Hall, Englewood Cliffs, N. J., 1973.

4. Alan Day, A characterization of modularity for congruence lattices of algebras, Canad. Math. Bull., 12 (1969), 167-173.

5. R. A. Dean and R. H. Oehmke, Idempotent semigroups with distributive right congruence lattices, Pacific J. Math., 14 (1964), 1187-1209.

6. Trevor Evans, The lattice of semigroup varieties, Semigroup Forum 2 (1971), 1-43.

7. B. Jónsson, Algebras whose congruence lattices are distributive, Math. Scand., 21 (1967), 110-121.

8. A. Malcev, On the general theory of algebraic systems, Mat. Sb. (N. S.), 35 (77) (1954), 3-20.

9. D. Papert, Congruence relations in semi-lattices, London Math. Soc., 39 (1964), 723-729.

10. E. T. Schmidt, Kongruenzrelationen Algebraischer Strukturen, Veb Deutscher Verlag, Berlin, 1969.

Received July 10, 1972.

California Institute of Technology

AND

UNIVERSITY OF HAWAII 


\section{PACIFIC JOURNAL OF MATHEMATICS}

\section{EDITORS}

RICHARD ARENS (Managing Editor) University of California

Los Angeles, California 90024

R. A. Beaumon'T

University of Washington Seattle, Washington 98105
J. Dugundj1*

Department of Mathematics University of Southern California Los Angeles, California 90007

D. Gilbarg and J. Milgram Stanford University Stanford, California 94305

\section{ASSOCIATE EDITORS}

E. F. BECKENBACH

B. H. NeUMANN

F. WOLF

K. YOSHIDA

\section{SUPPORTING INSTITUTIONS}

UNIVERSITY OF BRITISH COLUMBIA CALIFORNIA INSTITUTE OF TECHNOLOGY UNIVERSITY OF CALIFORNIA MONTANA STATE UNIVERSITY UNIVERSITY OF NEVADA NEW MEXICO STATE UNIVERSITY OREGON STATE UNIVERSITY UNIVERSITY OF OREGON OSAKA UNIVERSITY

\section{UNIVERSITY OF SOUTHERN CALIFORNIA STANFORD UNIVERSITY UNIVERSITY OF TOKYO UNIVERSITY OF UTAH WASHINGTON STATE UNIVERSITY UNIVERSITY OF WASHINGTON AMERICAN MATHEMATICAL SOCIETY NAVAL WEAPONS CENTER}

* C. R. DePrima California Institute of Technology, Pasadena, CA 91109, will replace J. Dugundji until August 1974. 


\section{Pacific Journal of Mathematics}

\section{Vol. 49, No. $1 \quad$ May, 1973}

A. Bigard, Free lattice-ordered modules ...........................

Richard Bolstein and Warren R. Wogen, Subnormal operators in strictly cyclic

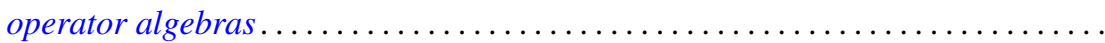

Herbert Busemann and Donald E. Glassco, II, Irreducible sums of simple

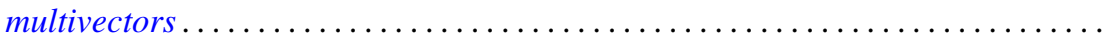

W. Wistar (William) Comfort and Victor Harold Saks, Countably compact groups

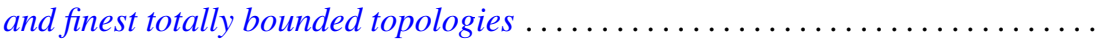

Mary Rodriguez Embry, Maximal invariant subspaces of strictly cyclic operator

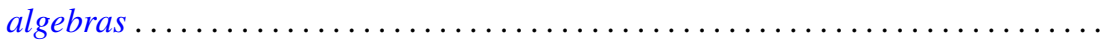

Ralph S. Freese and James Bryant Nation, Congruence lattices of semilattices......

Ervin Fried and George Grätzer, A nonassociative extension of the class of

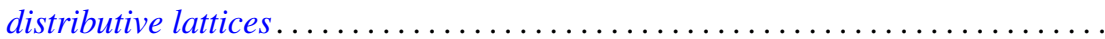

John R. Giles and Donald Otto Koehler, On numerical ranges of elements of locally

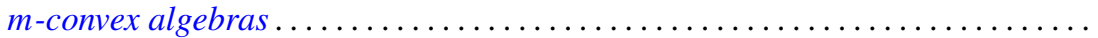

David A. Hill, On dominant and codominant dimension of $\mathrm{QF}-3$ rings ........ John Sollion Hsia and Robert Paul Johnson, Round and Pfister forms over $R(t) \ldots$ I. Martin (Irving) Isaacs, Equally partitioned groups . . . . . . . . . . . . . .

Athanassios G. Kartsatos and Edward Barry Saff, Hyperpolynomial approximation of solutions of nonlinear integro-differential equations.

Shin'ichi Kinoshita, On elementary ideals of $\theta$-curves in the 3-sphere and 2-links in the 4-sphere

Ronald Brian Kirk, Convergence of Baire measures

R. J. Knill, The Seifert and Van Kampen theorem via regular covering spaces ..

Amos A. Kovacs, Homomorphisms of matrix rings into matrix rings ..

Young K. Kwon, HD-minimal but no $H D$-minimal ..........

Makoto Maejima, On the renewal function when some of the mean renewal lifetimes are infinite

Juan José Martínez, Cohomological dimension of discrete modules over profinite groups.

W. K. Nicholson, Semiperfect rings with abelian group of units

Louis Jackson Ratliff, Jr., Three theorems on imbedded prime divisors of principal ideals.

Billy E. Rhoades and Albert Wilansky, Some commutants in $B(c)$ which are almost matrices

John Philip Riley Jr., Cross-sections of decompositions . . .

Keith Duncan Stroyan, A characterization of the Mackey uniformity $m\left(L^{\infty}, L^{1}\right)$ for

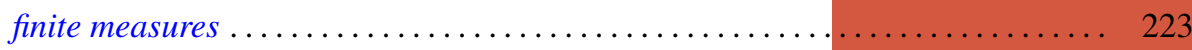

Edward G. Thurber, The Scholz-Brauer problem on addition chains . . . . . . . . . 229

Joze Vrabec, Submanifolds of acyclic 3-manifolds ............

Philip William Walker, Adjoint boundary value problems for compactified singular

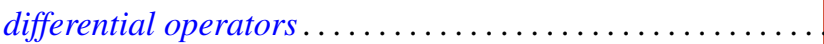

